Tohoku J. exp. Med., 1972, 107, 253-262

\title{
Cross-immunity among Allogeneic Tumors of Rats
}

\author{
Isamu Usubuchi, Yukio Sobajima, Hajime Kudo, Masa- \\ FUmi Kano and Tatsusuke Sato \\ Department of Pathology, ${ }^{*}$ Hirosaki University School of \\ Medicine, Hirosaki
}

\begin{abstract}
Usubuch, I., Sobajma, Y., Kudo, H., Kano, M. and Sato, T. Crossimmunity among Allogeneic Tumors of Rats. Tohoku J. exp. Med., 1972, 107 (3), 253-262 - Host resistance against the transplantation of allogeneic tumors, such as Yoshida sarcoma, Hirosaki sarcoma, Takeda sarcoma or Ascites Hepatoma 7974, occurs in rats immunized with allogeneic Usubuchi sarcoma. It is considered that allogeneic tumors of rats have common antigens and this antigenicity causes cross-immunity among various allogeneic tumors. The cross-immunity induced by the common antigens was usually weak as compared with the immunity demonstrated in the re-transplantation of the same tumor. In such a state, the antigenicity peculiar to the host, in which each tumor originated, acts effectively in addition to the common antigens. cross-immunity; allogeneic tumors
\end{abstract}

It was reported previously that rats, which had been cured of transplanted ascites tumors by the treatment with Mitomycin C, showed strong resistance against re-transplantation of other allogeneic tumors (Usubuchi 1964b). This suggested that cross-immunity was induced by the common antigens between allogeneic tumors. The cross-immunity was observed not only between tumors of rats but also between tumors of $\mathrm{C} 3 \mathrm{H} / \mathrm{He}$ mice (Usubuchi et al. 1966). On the basis of cross-immunity among tumors of rats and among those of mice, the inhibition of auto-transplantation of tumors was demonstrated in the primary autochthonous host immunized with allogeneic and syngeneic tumors (Usubuchi et al. 1967, 1969).

It has been an almost universal opinion of investigators in this field that crossimmunity between allogeneic or syngeneic tumors does not exist, since Klein et al. (1960) reported that cross-immunity could not inhibit the production of tumors. However, only strong cross-immunity has a possibility of inhibitory effect on the production of tumor. Therefore, the failure of inhibition of carcinogenesis does not necessarily deny the existence of cross-immunity. Prehn and Main (1957) confirmed the cross-immunity among methylcholanthrene-induced tumors in a certain degree. Recently, Zbar et al. (1969) found cross-reacting tumor-specific transplantation antigens (TSTA) in 3 of 8 diethylnitrosamine-induced strain-2 guinea pig hepatomas. Holmes et al. (1971) also reported that cross-reacting TSTA were demonstrated in 2 of 3 methylcholanthrene-induced strain-2 guinea pig sarcomas. This paper deals with the inhibition of intraperitoneal transplantation

Received for publication, November $30,1971$.

* Director: Prof. I. Usubuchi. 
of different allogeneic tumors as well as the same tumor by immunization with Usubuchi sarcoma (Usubuchi 1955) which regresses spontaneously in the subcutaneous transplantation into rats.

\section{Materials and Methods}

\section{Animals}

Non-inbred rats of both sexes, weighing from $150 \mathrm{~g}$ to $200 \mathrm{~g}$, were used.

\section{Tumors}

Usubuchi sarcoma (Usubuchi 1955): This tumor was established in the form of a transplantable ascites tumor converted from a methylcholanthrene-induced sarcoma of a non-inbred rat in our laboratory and has been transplanted serially since 1953 . The tumor inoculated subcutaneously in rats grows usually to the size of a thumb's head for a while and then regresses spontaneously in most cases.

Yoshida sarcoma (Yoshida 1949): This ascites tumor was found out as an ascites form originally in a non-inbred rat by Yoshida in 1943. The tumor was supplied by the Sasaki Institute, Tokyo.

Hirosaki sarcoma (Usubuchi 1956): This tumor having originated in 1951 as a spontaneous tumor of the cervical lymph node of a non-inbred rat was converted into an ascites form in our laboratory. Since then the serial intraperitoneal transplantation has been carried out. Biological and cytological properties of this tumor are very similar to those of Yoshida sarcoma.

Takeda sarcoma (Takeda 1952): This transplantable ascites tumor was supplied by the Department of Pathology, Hokkaido University. This tumor originated from a spontaneous tumor in the subcutaneous tissue of the chest of a non-inbred rat and was converted to a transplantable ascites tumor by Takeda in 1952. The characteristics of this tumor are very similar to those of Usubuchi sarcoma.

Ascites Hepatoma $79 \tau_{4}$ (Yoshida 195\%): This tumor was originally brought about in 1952 in the Sasaki Institute by intraperitoneal transplantation of DAB hepatoma in a non-inbred rat. The tumor used in the present experiments was supplied by the same institute.

\section{Immunization with Usubuchi sarcoma}

In every case, $10^{7}$ cells of Usubuchi sarcoma were inoculated subcutaneously and this immunization procedure was repeated 5 times at intervals of 4 to 5 days in the same host.

\section{Immunization with normal cells}

A part of control animals received 5 subcutaneous injections of $0.5 \mathrm{ml}$ of a freshly prepared $10 \%$ suspension of normal syngeneic liver cells at 4 - and 5 -day intervals.

Intraperitoneal challenges of various tumors in the rats immunized with Usubuchi sarcoma

The intraperitoneal inoculation of $10^{7}$ cells of Usubuchi sarcoma to the rats immunized with Usubuchi sarcoma as mentioned above was carried out as the 1st challenge. The ascites was daily examined. After the tumor was regarded as being cured, the 2nd challenge of Usubuchi sarcoma was carried out intraperitoneally in the same rat. Similarly, after cure of the formerly challenged tumor, $10^{7}$ cells of Yoshida sarcoma, Hirosaki sarcoma, Takeda sarcoma and AH 7974 were inoculated into the peritoneal cavity as the 3rd and 4th challenge. 


\section{Results}

Experiment 1. Challenges of Usubuchi sarcoma and Yoshida sarcoma to the rats immunized with Usubuchi sarcoma (Table 1)

The 1st challenge (Usubuchi sarcoma): In 11 of 14 immunized rats challenged with the 1st intraperitoneal transplantation of Usubuchi sarcoma, tumor cells vanished from the ascites on the next day, in 2 cases on the 2nd day, and in 1 case on the 3rd day. Twenty nonimmunized controls transplanted intraperitoneally with Usubuchi sarcoma died of the proliferation of tumor cells without exception.

TABLE 1. Results of intraperitoneal challenges of Usubuchi sarcoma and Yoshida sarcoma to the rats immunized with Usubuchi sarcoma

\begin{tabular}{|c|c|c|c|c|}
\hline \multirow{2}{*}{$\begin{array}{c}\text { Animal } \\
\text { No. }\end{array}$} & \multicolumn{4}{|c|}{ Period of existence of tumor cells after intraperitoneal challenges (days) } \\
\hline & $\begin{array}{c}\text { Challenge } 1 \\
\text { (Usubuchi sarcoma) }\end{array}$ & $\begin{array}{c}\text { Challenge } 2 \\
\text { (Usubuchi sarcoma) }\end{array}$ & $\begin{array}{c}\text { Challenge } 3 \\
\text { (Yoshida sarcoma) }\end{array}$ & $\begin{array}{c}\text { Challenge } 4 \\
\text { (Yoshida sarcoma) }\end{array}$ \\
\hline 1 & 0 & 0 & Tumor-death & - \\
\hline 2 & 2 & 0 & 0 & 1 \\
\hline 3 & 1 & 0 & Tumor-death & - \\
\hline 4 & 0 & 0 & 6 & 0 \\
\hline 5 & 0 & 0 & 0 & 0 \\
\hline 6 & 0 & 1 & 0 & 1 \\
\hline 7 & 0 & 0 & 0 & 0 \\
\hline 8 & 0 & 0 & 4 & 0 \\
\hline 9 & 0 & 0 & 4 & 0 \\
\hline 10 & 0 & 0 & 4 & 0 \\
\hline 11 & 0 & 0 & 0 & 0 \\
\hline 12 & 1 & 0 & 1 & 0 \\
\hline 13 & 0 & 0 & 1 & 0 \\
\hline 14 & 0 & 0 & 0 & 0 \\
\hline
\end{tabular}

The 2nd challenge (Usubuchi sarcoma): To 14 rats cured of the 1st challenge of Usubuchi sarcoma, the 2nd challenge of the same tumor was carried out. In 13 of 14 rats, tumor cells vanished on the next day, and in the rest on the 2 nd day. Twenty non-immunized controls transplanted intraperitoneally with Usubuchi sarcoma died of the proliferation of tumor cells without exception.

The 3rd challenge (Yoshida sarcoma): The rats cured of the 2nd challenge of Usubuchi sarcoma were inoculated with Yoshida sarcoma as the 3rd challenge. In 6 of 14 rats challenged with Yoshida sarcoma, tumor cells vanished on the next day, in 2 cases on the 2 nd day, in 3 cases on the 5 th day and in 1 case on the 7th day. The other 2 died of the proliferation of tumor cells. Twenty controls immunized with allogeneic liver cells and 20 non-immunized controls were transplanted intraperitoneally with Yoshida sarcoma. All of them died of the proliferation of tumor cells without exception.

The 4th challenge (Yoshida sarcoma): Yoshida sarcoma was challenged to 12 rats cured of the 3rd challenge of the same tumor. In 10 of these 12 cases, tumor cells vanished on the next day, and in the other 2 on the 2 nd day after the inocula- 
tion. Twenty non-immunized controls transplanted intraperitoneally with Yoshida sarcoma died of the proliferation of tumor cells without exception.

Experiment 2. Challenges of Usubuchi sarcoma and Hirosaki sarcoma to the rats immunized with Usubuchi sarcoma (Table 2)

The 1st challenge (Usubuchi sarcoma): The 1st challenge of Usubuchi sarcoma was carried out to 15 rats immunized with Usubuchi sarcoma by subcutaneous inoculation. In 7 cases of them, tumor cells vanished on the next day, in 2 cases on the $2 n d$ day, in 4 cases on the 3rd day, in 1 case on the 4th day, and in 1 case on the 5th day. Twenty non-immunized controls transplanted intraperitoneally with Usubuchi sarcoma died of the proliferation of tumor cells without exception.

TABLE 2. Results of intraperitoneal challenges of Usubuchi sarcoma and Hirosaki sarcoma to the rats immunized with Usubuchi sarcoma

\begin{tabular}{|c|c|c|c|c|}
\hline \multirow{2}{*}{$\begin{array}{l}\text { Animal } \\
\text { No. }\end{array}$} & \multicolumn{4}{|c|}{ Period of existence of tumor cells after intraperitoneal challenges (days) } \\
\hline & $\begin{array}{c}\text { Challenge } 1 \\
\text { (Usubuchi sarcoma) }\end{array}$ & $\begin{array}{c}\text { Challenge } 2 \\
\text { (Usubuchi sarcoma) }\end{array}$ & $\begin{array}{c}\text { Challenge } 3 \\
\text { (Hirosaki sarcoma) }\end{array}$ & $\begin{array}{c}\text { Challenge } 4 \\
\text { (Hirosaki sarcoma) }\end{array}$ \\
\hline 1 & 0 & 0 & 0 & 0 \\
\hline 2 & 0 & 0 & 0 & 0 \\
\hline 3 & 0 & 0 & 0 & 0 \\
\hline 4 & 2 & 0 & 1 & 0 \\
\hline 5 & 4 & 0 & 1 & 0 \\
\hline 6 & 2 & 1 & 0 & 0 \\
\hline 7 & 0 & 0 & 0 & 0 \\
\hline 8 & 0 & 1 & 1 & 0 \\
\hline 9 & 1 & 0 & 0 & 0 \\
\hline 10 & 1 & 0 & 0 & 0 \\
\hline 11 & 2 & 0 & 0 & 0 \\
\hline 12 & 0 & 0 & 0 & 0 \\
\hline 13 & 3 & 1 & 1 & 0 \\
\hline 14 & 0 & 0 & 0 & 0 \\
\hline 15 & 2 & 0 & 1 & 0 \\
\hline
\end{tabular}

The 2nd challenge (Usubuchi sarcoma): The 2nd challenge of Usubuchi sarcoma was carried out to 15 rats cured of the 1 st challenge of the same tumor. In 12 of these 15 cases, tumor cells vanished on the next day, and in 3 rats on the 2nd day. Twenty non-immunized controls transplanted intraperitoneally with Usubuchi sarcoma died of the tumor without exception.

The 3rd challenge (Hirosaki sarcoma): Hirosaki sarcoma was inoculated as the 3 rd challenge to 15 rats cured of the 2 nd challenge of Usubuchi sarcoma. In 10 of 15 cases, tumor cells vanished on the next day, and in the other 5 on the 2nd day. Twenty controls immunized with allogeneic liver cells and 20 non-immunized controls were transplanted intraperitoneally with Hirosaki sarcoma. All of them died of the tumor without exception.

The 4th challenge (Hirosaki sarcoma): Hirosaki sarcoma was inoculated as the 4th challenge to 15 rats cured of the 3rd challenge of the same tumor. In 
all of these 15 rats, tumor cells vanished on the next day of the inoculation. Twenty non-immunized controls transplanted intraperitoneally with Hirosaki sarcoma died of the proliferation of tumor cells without exception.

Experiment 3. Challenges of Usubuchi sarcoma and Takeda sarcoma to the rats immunized with Usubuchi sarcoma (Table 3)

The 1st challenge (Usubuchi sarcoma): The lst intraperitoneal transplantation of Usubuchi sarcoma was carried out to 17 rats immunized by subcutaneous inoculation of Usubuchi sarcoma. In 4 of these 17 cases, tumor cells vanished on the next day, in 1 case on the 2nd day, in 2 cases on the 3rd day, in 7 cases on the 5 th day, in 2 cases on the 7 th day' and in the other 1 on the 9th day after the inoculation. Twenty non-immunized controls transplanted intraperitoneally with Usubuchi sarcoma died of the tumor without exception.

TABLE 3. Results of intraperitoneal challenges of Usubuchi sarcoma and Takeda sarcoma to the rats immunized with Usubuchi sarcoma

\begin{tabular}{|c|c|c|c|c|}
\hline \multirow{2}{*}{$\begin{array}{l}\text { Animal } \\
\text { No. }\end{array}$} & \multicolumn{4}{|c|}{ Period of existence of tumor cells after intraperitoneal challenges (days) } \\
\hline & $\begin{array}{c}\text { Challenge } 1 \\
\text { (Usubuchi sarcoma) }\end{array}$ & $\begin{array}{c}\text { Challenge } 2 \\
\text { (Usubuchi sarcoma) }\end{array}$ & $\begin{array}{c}\text { Challenge } 3 \\
\text { (Takeda sarcoma) }\end{array}$ & $\begin{array}{c}\text { Challenge } 4 \\
\text { (Takeda sarcoma) }\end{array}$ \\
\hline 1 & 1 & 0 & 0 & 0 \\
\hline 2 & 4 & 0 & 0 & 0 \\
\hline 3 & 4 & 0 & 0 & 0 \\
\hline 4 & 6 & 0 & 0 & 0 \\
\hline 5 & 4 & 0 & 0 & 0 \\
\hline 6 & 4 & 0 & 0 & 0 \\
\hline 7 & 2 & 0 & 0 & 0 \\
\hline 8 & 0 & 0 & 0 & 0 \\
\hline 9 & 8 & 0 & 0 & 0 \\
\hline 10 & 0 & 0 & 1 & 0 \\
\hline 11 & 6 & 0 & 0 & 0 \\
\hline 12 & 4 & 0 & 0 & 0 \\
\hline 13 & 0 & 0 & 0 & 0 \\
\hline 14 & 0 & 0 & 0 & 0 \\
\hline 15 & 2 & 0 & 1 & 0 \\
\hline 16 & 4 & 1 & 0 & 0 \\
\hline 17 & 4 & 0 & 0 & 0 \\
\hline
\end{tabular}

The 2nd challenge (Usubuchi sarcoma): Usubuchi sarcoma was inoculated repeatedly as the 2 nd challenge to 17 rats cured of the 1st challenge of Usubuchi sarcoma. In 16 of these 17 cases, tumor cells vanished on the next day, and in the other 1 on the 2 nd day after the inoculation. Twenty non-immunized controls transplanted intraperitoneally with Usubuchi sarcoma died of the proliferation of tumor cells without exception.

The 3rd challenge (Takeda sarcoma): Takeda sarcoma was transplanted as the 3 rd challenge to 17 rats cured of Usubuchi sarcoma of the 2 nd challenge. In 15 of these 17 cases, tumor cells vanished on the next day, and in 2 cases on the 2nd day after the inoculation. Twenty controls immunized with allogeneic liver cells 
20 non-immunized controls transplanted intraperitoneally with Takeda sarcoma died of the proliferation of tumor cells without exception.

The 4th challenge (Takeda sarcoma): Takeda sarcoma was transplanted as the 4th challenge to 17 rats cured of the $3 \mathrm{rd}$ challenge of the same tumor. In all cases, tumor cells vanished on the next day. Twenty non-immunized controls transplanted intraperitoneally with Takeda sarcoma died of the tumor without exception.

Experiment 4. Challenges of Usubuchi sarcoma and $A H 7974$ to the rats immunized with Usubuchi sarcoma (Table 4)

The 1st challenge (Usubuchi sarcoma): The 1st challenge of Usubuchi sarcoma was carried out to rats immunized by subcutaneous inoculation of Usubuchi sarcoma. In 8 of these 15 cases, tumor cells vanished on the next day, in 5 cases on the 2 nd day, and in the other 2 on the 3rd day after the inoculation. Twenty non-immunized controls transplanted with Usubuchi sarcoma intraperitoneally died of the proliferation of tumor cells without exception.

TABLE 4. Results of intraperitoneal challenges of Usubuchi sarcoma and AH 7974 to the rats immunized with Usubuchi sarcoma

\begin{tabular}{|c|c|c|c|c|}
\hline \multirow{2}{*}{$\begin{array}{l}\text { Animal } \\
\text { No. }\end{array}$} & \multicolumn{4}{|c|}{ Period of existence of tumor cells after intraperitoneal challenges (days) } \\
\hline & $\begin{array}{c}\text { Challenge } 1 \\
\text { (Usubuchi sarcoma) }\end{array}$ & $\begin{array}{c}\text { Challenge 2 } \\
\text { (Usubuchi sarcoma) }\end{array}$ & $\begin{array}{r}\text { Challenge } 3 \\
\text { (AH 7974) }\end{array}$ & $\begin{array}{r}\text { Challenge } 4 \\
\text { (AH 7974) }\end{array}$ \\
\hline 1 & 0 & 0 & 1 & 0 \\
\hline 2 & 0 & 0 & Tumor-death & - \\
\hline 3 & 0 & 0 & Tumor-death & - \\
\hline 4 & 0 & 0 & 1 & 1 \\
\hline 5 & 1 & 0 & Tumor death & - \\
\hline 6 & 2 & 0 & 4 & 1 \\
\hline 7 & 1 & 0 & Tumor-death & - \\
\hline 8 & 0 & 0 & Tumor-death & - \\
\hline 9 & 1 & 0 & 14 & 2 \\
\hline 10 & 1 & 0 & Tumor-death & $二$ \\
\hline 11 & 1 & 0 & 4 & 1 \\
\hline 12 & 0 & 0 & 2 & 1 \\
\hline 13 & 2 & 1 & Tumor-death & - \\
\hline 14 & 0 & 0 & 4 & 1 \\
\hline 15 & 0 & 0 & Tumor-death & $二$ \\
\hline
\end{tabular}

The 2nd challenge (Usubuchi sarcoma): Usubuchi sarcoma was transplanted as the 2 nd challenge to 15 cases cured of the lst challenge of the same tumor. In 14 of these 15 cases, tumor cells vanished on the next day, and in the other 1 on the 2nd day after the inoculation. Twenty non-immunized controls transplanted intraperitoneally with Usubuchi sarcoma died of the proliferation of tumor cells without exception.

The 3rd challenge ( $A H$ 7974): The inoculation of $\mathrm{AH} 7974$ was carried out as the 3 rd challenge to 15 rats cured of Usubuchi sarcoma of the 2 nd challenge. In 
2 of these 15 cases, tumor cells vanished on the 2nd day, in 1 case on the 3rd day, in 3 cases on the 5th day, and in 1 case on the 15th day after the inoculation. The other 8 died of the proliferation of tumor cells. Twenty controls immunized with allogeneic liver cells and 20 non-immunized controls were transplanted intraperitoneally with $\mathrm{AH} \mathrm{7974.} \mathrm{All} \mathrm{of} \mathrm{them} \mathrm{died} \mathrm{of} \mathrm{the} \mathrm{proliferation} \mathrm{of} \mathrm{tumor}$ cells without exception.

The 4th challenge ( $A H$ 7974): $\mathrm{AH} 7974$ was transplanted as the 4th challenge to 7 rats cured of the 3 rd challenge of the same tumor. In 1 case, tumor cells vanished on the next day, in 5 cases on the 2 nd day, and in the other 1 on the 3rd day after the inoculation. Twenty non-immunized controls transplanted intraperitoneally with $\mathrm{AH} 7974$ died of the proliferation of tumor cells without exception.

\section{Discussion}

The results of transplantation of Usubuchi sarcoma as the 1st challenge to the rats immunized subcutaneously with the same tumor showed that the proliferation of the tumor was inhibited, but its degree was not always the same. Namely, in Exp. 1 and Exp. 4, the inhibition of proliferation of the tumor was strong, while in Exp. 2 and Exp. 3 the inhibition was relatively weak and the tumor cells remained for several days in many cases. The fact that the degree of inhibition of the intraperitoneal transplantation of Usubuchi sarcoma was not always constant, seems to indicate that the immunity by means of the repeated subcutaneous injection of Usubuchi sarcoma was not enough to inhibit fully the intraperitoneal proliferation of the tumor.

The intraperitoneal transplantation of the same tumor as the 2nd challenge was inhibited well in all experimental groups. This suggests the production of sufficient immunity against Usubuchi sarcoma after the lst challenge.

As the 3rd challenge, the intraperitoneal transplantation of allogeneic tumors, such as Yoshida sarcoma, Hirosaki sarcoma, Takeda sarcoma and AH 7974, was carried out to rats immunized sufficiently against Usubuchi sarcoma. The results that the inhibition of the transplantation was observed usually in all experimental groups suggest that cross-immunity was established owing to the existence of common antigens among tumor cells of allogeneic animals. However, its degree was not always definite. That is to say, challenges of Hirosaki sarcoma and Takeda sarcoma were inhibited almost completely, while in cases of Yoshida sarcoma and $\mathrm{AH} 7974$, the inhibition was not so strong. These data seem to show that the cross-immunity could not inhibit the allogeneic tumor completely, because each tumor has the peculiar antigenicity (including histocompatibility or transplantation antigens) in addition to the common antigens. In other words, the transplantation of Hirosaki sarcoma and Takeda sarcoma was inhibited by mere cross-immunity on account of the weak proliferation of these tumors. On the other hand, the transplantation of Yoshida sarcoma and AH 7974 was not 
inhibited completely by mere cross-immunity when the proliferation of the tumors was strong.

Strong immunity recognized in the 4th challenge of the same tumor as the 3rd challenge may be a natural outcome of the same antigenicity.

The results of the above four groups of experiments seem to indicate that the common antigens exist between Usubuchi sarcoma and other tumors tested, such as Yoshida sarcoma, Hirosaki sarcoma, Takeda sarcoma and AH 7974. The cross-immunity demonstrated in these experiments may be explained only by the existence of the common antigens.

In view of the results so far obtained, it would be reasonable to assume that common antigenicity exists among all tumors of rats not yet tested. This common antigenicity is supposed to exist in autochthonous tumors per se (Usubuchi 1964). Furthermore, it may be expected that this common antigenicity among all allogeneic tumors is contained in allogeneic immature cells, such as those of the bone marrow, lymph node, testis or placenta as well as tumor cells (Usubuchi 1964a, 1956).

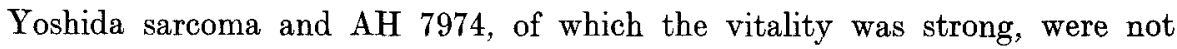
completely inhibited by cross-immunity, while Hirosaki sarcoma and Takeda sarcoma, of which the vitality was weak, were inhibited completely by mere crossimmunity. The data mentioned above may be explained satisfactorily by the supposition that antigens in each tumor cell are composed of antigens common to allogeneic tumors and those peculiar to each tumor (including histocompatibility or transplantation antigens). This peculiar antigenicity of each tumor can be thought to be caused by the tumor cell itself. But, under consideration of antitransplantation immunity showing marked differences between autochthonous tumor and transplanted tumors, it would be more reasonable that the peculiar antigenicity is caused by individual variation of the primary autochthonous host.

TABLE 5. Anti-transplantation immunity by means of a variety of experimental methodology

\begin{tabular}{|c|c|c|c|c|c|c|}
\hline \multicolumn{2}{|c|}{$\begin{array}{l}\text { Exp. } \\
\text { group }\end{array}$} & $\begin{array}{l}\text { Group } \\
\text { No. }\end{array}$ & $\begin{array}{c}\text { Immunizing } \\
\text { tumors }\end{array}$ & $\begin{array}{l}\text { Challenging } \\
\text { tumors }\end{array}$ & Host & $\begin{array}{l}\text { Antigens acting in } \\
\text { anti-transplanta- } \\
\text { tion immunity }\end{array}$ \\
\hline \multirow{2}{*}{$\begin{array}{l}\text { Immunizing } \\
\text { and } \\
\text { challenging } \\
\text { tumors of } \\
\text { identical } \\
\text { origin }\end{array}$} & $\begin{array}{l}\text { Autochthonous } \\
\text { tumor }\end{array}$ & 1 & A & A & $\alpha$ & \\
\hline & $\begin{array}{l}\text { Allogeneic } \\
\text { tumor }\end{array}$ & $\begin{array}{l}2 \\
3\end{array}$ & $\begin{array}{l}\mathrm{B} \\
\mathrm{C}\end{array}$ & $\begin{array}{l}\mathrm{B} \\
\mathrm{C}\end{array}$ & $a$ & $\frac{b}{c}$ \\
\hline \multicolumn{2}{|c|}{$\begin{array}{l}\text { Immunizing and } \\
\text { challenging tumors } \\
\text { of different origin }\end{array}$} & $\begin{array}{l}4 \\
5\end{array}$ & $\begin{array}{l}\mathrm{B} \\
\mathrm{B}\end{array}$ & C & $\begin{array}{l}\alpha \\
\alpha\end{array}$ & \\
\hline
\end{tabular}


On the basis of this hypothesis, anti-transplantation immunity according to a variety of methodology may be explained as shown in Table 5. Group 1 shows the immunity of the primary autochthonous tumor, which was proved by Klein et al. (1960), Takeda et al. (1966) and Usubuchi et al.(1962). Common antigens, which exist among allogeneic and syngeneic tumors, are considered to exist in the primary autochthonous tumor itself. In Groups 2 and 3, the immunity on the occasion of re-transplantation of the same tumor is explained. As the peculiar antigenicity of each tumor is added to the common antigens, the expression of the immunity is demonstrated more strongly. Group 4 shows cross-immunity due to the common antigens, which has been proved in the experiments of this paper. Group 5 shows the inhibition of autochthonous transplantation of primary tumor by the common antigens as reported previously by us (Usubuchi et al. 1967, 1969). Usubuchi (1956) reported some inhibitory effect of cross-immunity on methylcholanthrenecarcinogenesis, using tumor tissues and normal immature cells as antigens.

\section{References}

1) Holmes, E.C., Morton, D.L., Schidlovsky, G. \& Trahan, E. (1971) Cross-reacting tumor-specific transplantation antigens in methylcholanthrene-induced guinea pig sarcomas. J. nat. Cancer Inst., 46, 693-700.

2) Klein, G., Sjögren, H.O., Klein, E. \& Hellström, K.E. (1960) Demonstration of resistance against methylcholanthrene-induced sarcomas in the primary autochtho. nous host. Cancer Res., 20, 1561-1572.

3) Prehn, R.T. \& Main, J.M. (1957) Immunity to methylcholanthrene-induced sarcomas. J. nat. Cancer Inst., 18, 769-778.

4) Takeda, K., Aizawa, M., Iwamura, T., Sasage, S., Mastumoto, K. \& Kanehira, S. (1952) On the nature of a new ascites tumor of rat (Takeda) and its relation to ascites sarcoma of Yoshida, MTK 1-2 and Hirosaki types. Gann, 43, 132-135.

5) Takeda, K., Aizawa, M., Kikuchi, Y., Yamawaki, S. \& Nakamua, K. (1966) Tumor autoimmunity against methylcholanthrene-induced sarcomas of the rat. Gann, 57, $221-240$.

6) Usubuchi, I. (1955) Studies on the pathology of the Usubuchi sarcoma. Acta path. jap. 5 (Suppl.), 395-407.

7) Usubuchi, I. \& Abe, H. (1956) The Hirosaki sarcoma. A lymphosarcomatosis of the rat. Gann, 47, 117-128.

8) Usubuchi, I. (1956) Inhibitory effect of immunization on production of tumor. Gann, 47, 534-535.

9) Usubuchi, I., Sobajima, Y. \& Hongo, T. (1962) Resistance against methylcholanthrene-induced sarcomas in the autochthonous host. Proc. Jap. Cancer Ass., 21st Ann. Meeting (Jap.), 286.

10) Usubuchi, I. (1964a) Immunity to cancer. Naika (Jap.), 13, 1025-1030.

11) Usubuchi, I. (1964b) Administration method of Mitomycin C. Acta Uu. int. Cancr. 20, $317-320$.

12) Usubuchi, I., Sobajima, Y. \& Kudo, H. (1966) Cross-immunity among C3H mouse tumors. Nipponrinsho (Jap.), 24 (Suppl.), 113-117.

13) Usubuchi, I., Sobajima, Y., Kudo, H., Hongo, T. \& Sugawara, M. (1967) Inhibitory effect of cross-immunity on autotransplantation of $\mathrm{C} 3 \mathrm{H}$ mouse mammary carcinomas. Igakunoayumi (Jap.), 61, 692-696.

14) Usubuchi, I., Sobajima, Y., Kudo, H., Uchiyama, T., Shichinohe, H. \& Toda, S. (1969) Inhibitory effect of cross-immunity on autotransplantation of rat methylcholanthrene-induced tumors. Igakunoayumi (Jap.), 69, 672-673. 
15) Yoshida, T. (1949) The Yoshida sarcoma, an ascites tumor. Gann, 40, 1-21.

16) Yoshida, T. (1957) Studien über das Ascites-Hepatom. Zugleich ein Beitrag zum Begriff der cellulären Autonomie im Wachstum der malignen Geschwulst einerseits, und der Individualität der einzelnen Geschwulst andererseits. Virchows Arch. path. Anat. 330, 85-105.

17) Zbar, B., Wepsic, H.T., Rapp, H.J., Borsos, T., Kronman, B.S. \& Churchill, W.H. Jr. (1969) Antigenic specificity of hepatomas induced by strain-2 guinea pigs by diethylnitrosamine. J. nat. Cancer Inst., 43, 833-841. 